\title{
VULNERABILITÄTSERFAHRUNGEN UND DIE ERARBEITUNG VON AGENCY: ANKOMMENSPROZESSE JUNGER GEFLÜCHTETER
}

Rebecca Mörgen und Peter Rieker

Universität Zürich, Institut für Erziehungswissenschaft

E-Mail: rmoergen@ife.uzh.ch

URL: https://www.ife.uzh.ch/de/research/abe/mitarbeitende2/moergenrebecca.html

Universität Zürich, Institut für Erziehungswissenschaft

E-Mail: prieker@ife.uzh.ch

URL: https://www.ife.uzh.ch/de/research/abe/mitarbeitende2/riekerpeter.html

Zitationsvorschlag:

Mörgen, Rebecca/Rieker, Peter (2021): Vulnerabilitätserfahrungen und die Erarbeitung von Agency: Ankommensprozesse junger Geflüchteter. In: Gesellschaft - Individuum Sozialisation (GISo). Zeitschrift für Sozialisationsforschung, 2 (1). DOI:

10.26043/GISo.2021.1.3

Link zum Artikel:

https://doi.org/10.26043/GISo.2021.1.3 


\title{
VULNERABILITÄTSERFAHRUNGEN UND DIE ERARBEITUNG VON AGENCY: ANKOMMENSPROZESSE JUNGER GEFLÜCHTETER ${ }^{1}$
}

\author{
Rebecca Mörgen und Peter Rieker
}

\begin{abstract}
Unbegleitete minderjährige Geflüchtete befinden sich nach ihrer Ankunft in Europa in prekären Lebenssituationen, die mit Vulnerabilitätserfahrungen einhergehen, die sie gleichzeitig bearbeiten und bewältigen. Denn mit der Ankunft ist zwar die Fluchtmigration beendet, doch angekommen sind die Jugendlichen noch nicht. Wie gestalten Jugendliche den Prozess des Ankommens, und welche Formen von Agency erarbeiten sie sich unter den jeweiligen sozialen Bedingungen? Diese Fragen fokussiert der Beitrag und rekonstruiert auf Basis von qualitativen Interviews mit jungen Geflüchteten, wie sie Vulnerabilitätserfahrungen und Agency in Bezug auf ihren Ankommensprozess thematisieren.
\end{abstract}

Keywords: Agency, Vulnerabilität, unbegleitete minderjährige Geflüchtete, Ankommensprozess, Fluchtmigration

Mit der Ankunft in europäischen Ländern, wie z. B. der Schweiz, und dem Stellen eines Asylgesuchs ist für unbegleitete minderjährige Geflüchtete (im Folgenden mineurs non accompagnés: MNA) zwar die Fluchtmigration beendet, doch von einem "Angekommen-Sein“ im Aufnahmekontext kann nicht ausgegangen werden. ${ }^{2}$ Vielmehr wird auf die mit der Ankunft beginnenden Herausforderungen und Ambivalenzen der Lebenssituationen der MNA hingewiesen: Ihr Alltag sei durchgängig von „Unsicherheiten geprägt" (Lechner et. al. 2017, 18) und sie bewegen sich in einem Spannungsverhältnis zwischen "Vulnerabilität und Eigenmotivation" sowie in „Kontakttreten und Anderssein“ (Findening/Klinger 2019) in Bezug auf die sie aufnehmende Mehrheitsgesellschaft. Die Ankunft werde als angsteinflössend wahrgenommen (Detemple 2016, 57) und das Erleben strukturell bedingter Einschränkungen, bspw. durch das Asylsystem, führe zu Gefühlen der Ohnmacht und Hilflosigkeit (Hargasser 2015, 124). Diese Betrachtungsweise verbindet die Lebenssituationen von jungen Geflüchteten implizit mit einer
Schutzwürdigkeit und Unterstützungsnotwendigkeit, die ihnen ausschliesslich eine vulnerable Position zuschreibt.

Die Jugendlichen befinden sich also auch nach der Ankunft in Europa in prekären Lebenssituationen, die mit Vulnerabilitätserfahrungen einhergehen, die sie aber auch bewältigen und bearbeiten. Allerdings fehlt es an Erkenntnissen dazu, wie geflüchtete Jugendliche ihr Leben, den damit verbundenen Prozess des Ankommens und die verschiedenen (institutionellen) Übergänge gestalten, wahrnehmen und erleben (Zeller et al. 2020) und welche Möglichkeitsräume sich unter den jeweiligen sozialen Bedingungen für sie ergeben können.

In der Schweiz, auf die sich die Überlegungen des vorliegenden Beitrags beziehen, werden MNA nach ihrer Ankunft in unterschiedlichen Kontexten institutionell untergebracht und betreut, wie bspw. in spezifischen MNA-Einrichtungen, in Pflegefamilien oder Gemeinschaftsunterkünften (Rieker et al. 2021). Es kann davon ausgegangen werden, dass sie während dem

\footnotetext{
${ }^{1}$ Wir möchten an dieser Stelle ganz herzlich Alex Knoll, Anne Caroline Ramos, Anna Schnitzer und den anonymen Gutachter*innen für die anregenden und konstruktiven Hinweise zu Vorversionen dieses Textes danken.

2 In der Schweiz wird der Begriff „mineurs non accompagnés“ (MNA) verwendet. Im Unterschied zu dem Begriff der „unbegleiteten minderjährigen Asylsuchenden“ umfasst dieser alle Kinder und Jugendlichen, die ohne Sorgeberechtigte in der Schweiz leben, und bezieht sich nicht nur auf den Status des Asylverfahrens.
} 
Ankommen unterschiedliche institutionelle Übergangs- und damit verbundene Diskontinuitätserfahrungen machen, wie sie aus der CareLeaving-Debatte bekannt sind (vgl. Göbel et al. 2020).

Vor diesem Hintergrund fokussiert der Beitrag die Frage, wie Jugendliche mit Fluchterfahrung den Prozess des Ankommens in der Schweiz erleben und gestalten, um vor diesem Hintergrund die sozialen Bedingungen von Vulnerabilität als auch Agency zu reflektieren. Es wird untersucht, inwiefern sich die Jugendlichen in Bezug auf den Ankommensprozess unter Berücksichtigung der jeweiligen sozialen und kontextspezifischen Bedingungen Handlungsmöglichkeiten erarbeiten. Zudem wird danach gefragt, welche Möglichkeitsräume sich für sie eröffnen und welche sozialen Beziehungen sie relevant setzen. Hierfür wird sich auf Interviews bezogen, die mit geflüchteten Jugendlichen im Kontext des Projekts „Unbegleitete minderjährige Geflüchtete in institutioneller Betreuung: Chancen und Herausforderungen“" geführt wurden. ${ }^{3}$

Im Folgenden werden zunächst der Forschungsund Diskussionsstand zu Verwundbarkeit und Agency in der Fluchtmigrationsforschung skizziert (1), bevor auf method(olog)ische Überlegungen des Projekts eingegangen wird (2). Im Anschluss daran werden zwei Fälle präsentiert, in denen die Ankunft in der Schweiz in spezifischer Weise erlebt wird, wobei biographische Dimensionen einen bedeutsamen Stellenwert haben und unterschiedliche Bedingungen der Ermöglichung wie auch Verhinderung von Agency und Vulnerabilitätserfahrungen deutlich werden (3). Den Abschluss bildet ein kurzes Fazit (4).

\section{FORSCHUNGS- UND DISKUSSIONSSTAND: VERWUNDBARKEIT UND AGENCY VON MNA IN DER FLUCHTMIGRATIONSFORSCHUNG}

Migrations- und Fluchtbewegungen sowie damit einhergehende biografische Prozesse werden seit den 90er-Jahren in der Fluchtmigrationsforschung zu jungen Geflüchteten mit Fragen von
Vulnerabilität und Handlungsfähigkeit verknüpft. Dies hat zunächst damit zu tun, dass junge Geflüchtete routinemässig - bspw. durch das Büro des Hohen Kommissars für Flüchtlinge der Vereinten Nationen (UNHCR) oder die EU-Rechtsprechung - in öffentlichen Diskursen als vulnerable Personengruppe kategorisiert werden. Eine besondere Rolle spielt hierbei die Darstellung der MNA als traumatisierte, verletzliche und schutzbedürftige Kinder (Lems et al. 2019). In dieser Adressierungsweise als vulnerables Subjekt befinden sich junge Geflüchtete ausserhalb dessen, was in europäischen Konzepten als "normale" und „ideale“ Kindheit betrachtet wird (Wernesjö 2012, 504) - wobei sich eurozentrische Konzeptionen von Kindheit eher durch Abhängigkeit und Schutzbedürftigkeit als durch Prozesse der Autonomisierung auszeichnen (Liebel 2017, 69). Dies sei zum einen der Fall, weil innen eine "normale" Kindheit vorenthalten werde (Wernesjö 2012, 504) und sie mitunter als Opfer verantwortungsloser Eltern gelten, die ihre Abhängigkeit ausnutzen und sie ungeachtet ihrer kindlichen Vulnerabilität auf die Flucht schicken. Zum anderen wird die Ausgeglichenheit und Gleichmütigkeit im Auftreten minderjähriger Geflüchteter als Fassade gesehen, hinter der Traumata, Ängste und Schuldgefühle ausgemacht werden (Kurz-Adam 2016, 43). Psychische Vulnerabilität und Hilflosigkeit werden somit mit traumatisierenden Verlusten und Verfolgungserfahrungen im Heimatland und auf der Flucht begründet (ebd.). Zudem werden Vulnerabilitätserfahrungen im Rahmen vorliegender Studien auf verschiedene kontextspezifische Bedingungen zurückgeführt: Unzureichende Partizipationsmöglichkeiten im Asylverfahren (Hargasser 2016), eine fürsorgliche Betreuung, die Selbstständigkeit verhindere (Jurt/Roulin 2016), fehlende Vertrauens- und Zukunftskonstellationen, die die jeweilige Handlungsmächtigkeit bedrohen (Zeller et al. 2020, 222) oder prekäre, instabile Beziehungen, die das Gefühl, "wirklich angekommen zu sein" (Lechner et. al. 2017, 18), verhinderten.

Dem gegenüber konzentriert man sich an anderer Stelle auf Fragen der Bedeutung wie auch

\footnotetext{
${ }^{3}$ Das Forschungsprojekt wird seit 2018 am Institut für Erziehungswissenschaft der Universität Zürich durchgeführt. Es wird vom Schweizerischen Nationalfonds gefördert und ist Teil des Nationalen Forschungsprogramms NFP 76. Im Projekt arbeiten Ellen Höhne, Rebecca Mörgen und Peter Rieker (Projektleitung).
} 
Herstellung von Agency junger Geflüchteter, wobei spezifische Erfahrungen im Herkunftskontext untersucht werden, wie z. B. die Übernahme von Verantwortung für andere Familienmitglieder und das Agieren als Haushaltsvorstand (Payne 2012). Zudem gelten junge Geflüchtete teilweise auch im familialen Kontext als autonome Akteur*innen, die ihre Fluchtentscheidung nicht von familialer Unterstützung abhängig machen (Heidbrinck/Statz 2017, 547 ff.; Belloni 2019). Andere Studien wiederum konzentrieren sich auf die Bedeutung von Handlungsmacht junger Geflüchteter im europäischen Grenzregime; hier werden z. B. ihre Verhandlungen mit Mitarbeitenden in Flüchtlingsunterkünften skizziert, in deren Verlauf sie ihre eigenen Interessen und Vorstellungen kompetent vertreten (Otto 2019).

Auf die Wirkmächtigkeit des Diskurses, der MNA auf essentialisierende Art und Weise als unterstützungs- und hilfsbedürftig kategorisiert und positioniert (Clark 2007), wird in verschiedenen empirischen Studien kritisch hingewiesen. Denn die Konstruktion der MNA als vulnerable Subjekte ruft in der Regel eine Bearbeitungskultur auf den Plan, z. B. die Besonderheiten der institutionellen Betreuung, die als Bearbeitungsversuche von Vulnerabilität gelesen werden können, und die die Legitimität von Sozialpädagogik in Bezug auf Betreuung und Unterbringung begründet. Somit durchdringe der Diskurs bspw. auch die institutionellen Kontexte der Unterstützungsangebote, in denen geflüchtete Jugendliche dazu aufgefordert werden, sich selbst als verletzlich und unterstützungsbedürftig zu zeigen, um überhaupt Zugang zu den entsprechenden Ressourcen zu erhalten (Otto 2019; Utas 2005). Diese Selbstpositionierungen bilden aber gleichzeitig die Grundlage für die Legitimitätsansprüche innerhalb des Asylsystems. Denn Vulnerabilität symbolisiere eine „Währung“ (Lems et al. 2019, 326), die eingesetzt werden müsse, um die Gegenleistung, wie Zugang zu gesellschaftlichen Ressourcen in Form von institutioneller Unterstützung oder Bildungsangebote, erhalten zu können. Und solch eine Selbstrepräsentation als vulnerabel kann ihrerseits als spezifische Form von Agency angesehen werden.
Vor diesem Hintergrund betonen bspw. Mats Utas (2005) mit dem Begriff "Victimcy“ oder Laura Otto (2019) mit "adult minors“ die Einnahme einer relationalen Perspektive auf das Verhältnis von Agency und Vulnerabilität, die den sozialen, ökonomischen, politischen und kontextspezifischen Bedingungen der Lebensführungsweisen der jungen Geflüchteten Beachtung schenkt (vgl. auch: Clark 2007; Belloni 2019). Sie verweisen auf die paradoxale Verbindung zwischen einer Selbst- und Fremdpositionierung und machen darauf aufmerksam, dass die Selbstpräsentation der Jugendlichen innerhalb des Migrationsregimes als vulnerabel, gleichzeitig aber auch als Ausdruck der Herstellung einer Handlungsfähigkeit und Handlungsmacht innerhalb des Asylsystems gelesen werden kann. Die jeweiligen Positionierungen in den Interaktionen mit institutionellen Akteur*innen unterliegen immer wieder alltäglichen Aushandlungen und entziehen sich einer eindeutigen Zuordnung eines handlungsfähigen oder vulnerablen Subjektstatus.

Mit dieser Einordnung in den Forschungsstand knüpft der Beitrag an konzeptionelle-theoretische Überlegungen zu einer relationalen Betrachtungsweise von Vulnerabilität und Agency an, die diese nicht als eine individuelle Eigenschaft - im Sinne einer Wesenszuschreibung konzipieren (Burkitt 2015; MacKenzie et al. 2013). Vielmehr gehen wir davon aus, dass sich Agency und Vulnerabilität situativ in sozialen Prozessen konstituieren und es sich um subjektive Konzeptionen von Agency- als auch Vulnerabilitätserfahrungen handelt (Helfferich 2020). Gemeinsam ist diesen Perspektiven die Verortung und Herstellung verschiedener Dimensionen von Agency innerhalb sozialer Beziehungskonstellationen wie auch sozialer Bedingungen, die für die Jugendlichen besonders relevant sind, sofern von einer existentiellen sozialen Angewiesenheit als auch Verwiesenheit auf Andere ausgegangen wird (Eßer/Schröder 2020). Damit wird von einem Subjektverständnis ausgegangen, das, mit Bernhard Waldenfels (2002) gesprochen, durch „Bruchlinien der Erfahrung" gekennzeichnet ist, zu denen es sich verhält, auf die es reagiert und antwortet, in denen Vulnerabilität ein Grundmoment von Erfahrungen darstellt (Stöhr 2019, 161) und in denen sich Möglichkeitsräume eröffnen. Die kontext- 
und situationsspezifischen Erfahrungen der Jugendlichen lassen sich sodann als eine Eröffnung und Begrenzung der Handlungsfähigkeiten und -möglichkeiten wie auch der Handlungsohnmacht verstehen. Es wird zu zeigen sein, wie sich geflüchtete Jugendliche Agency unter prekären Bedingungen des Migrationsregimes und des Ankommens in dem Aufnahmekontext erarbeiten und inwiefern mit spezifischen Vulnerabilitätserfahrungen begrenzende als auch ermöglichende Möglichkeitsräume einhergehen können.

\section{METHODISCHES VORGEHEN UND ME- THODOLOGISCHE ÜBERLEGUNGEN ZU DER SELBSTREPRÄSENTATION VON MNA IM PROJEKTKONTEXT}

Überlegungen zum Prozess des Ankommens sowie die Frage danach, welche sozialen Bedingungen und Beziehungen wie von geflüchteten Jugendlichen in den unterschiedlichen Phasen der Übergangs- und Diskontinuitätserfahrungen thematisiert werden, werden mit Bezug auf die Studie „Unbegleitete minderjährige Geflüchtete in institutioneller Betreuung: Chancen und Herausforderungen" empirisch analysiert und diskutiert. Das Projekt untersucht die (institutionelle) Unterbringung und Betreuung von MNA, wobei auf das Spannungsverhältnis von Fürsorge und Zwang fokussiert wird. Im Zuge dessen wird in unterschiedlichen institutionellen Betreuungskontexten der deutschsprachigen Schweiz (MNA-Zentren, Durchgangszentren, Pflegefamilien) über einen Zeitraum von bis zu drei Monaten an dem Alltag der Betreuung teilgenommen und ethnographisch beobachtet. Zudem werden Interviews mit den verschiedenen am Alltag der Betreuung Beteiligten (Jugendliche, Fachkräfte, Pflegeeltern) geführt.

Dieser Beitrag bezieht sich ausschliesslich auf die im Rahmen der Studie bisher durchgeführten Interviews mit elf Jugendlichen und fokussiert damit deren subjektive Perspektiven. Insgesamt wurden zehn männliche Jugendliche und eine junge Frau interviewt, die mindestens seit einem Jahr in der Schweiz leben, ganz unterschiedliche soziale Hintergründe aufweisen, aus unterschiedlichen Ländern (v. a. Eritrea, Afghanistan, Syrien) in die Schweiz migriert sind und entwe- der in einer Heimeinrichtung oder in einer Pflegefamilie leben. Der Zugang zu Jugendlichen, die in institutionellen Betreuungskontexten, wie MNAHeimen und Gemeinschaftsunterkünften, untergebracht sind, erfolgte auf Basis der ethnographischen Feldaufenthalte durch die jeweilige Forscherin. Die in den Einrichtungen lebenden Jugendlichen wurden nach einer gewissen Zeit der Teilnahme am Alltag, der damit verbundenen Kontaktaufnahme und des Beziehungsaufbaus von den Forscherinnen persönlich für die Durchführung des Interviews angesprochen. Im Unterschied dazu erfolgte der Zugang zu denjenigen Jugendlichen, die in Pflegefamilien untergebracht sind, über eine Pflegefamilienplatzierungsorganisation. Im Anschluss an ein Vorgespräch mit den Pflegefamilienmitgliedern wurden Interviews mit den Jugendlichen als auch den Pflegeeltern geführt. Die Interviews mit den Jugendlichen fanden an von ihnen selbstgewählten Orten und somit in Restaurants, Cafés, während einem Spaziergang, in den Klassenzimmern der Einrichtungen oder bei ihnen zuhause statt.

Methodisch orientieren wir uns am Verfahren des problemzentrierten Interviews, d. h. den Gesprächen liegt ein Leitfaden zugrunde, mit dem jedoch je nach Interviewsituation flexibel umgegangen wird. Gleichzeitig wurde versucht, möglichst wenige Vorgaben zu machen, um den interviewten Jugendlichen zu ermöglichen, eigene Relevanzsetzungen vorzunehmen (Witzel 2000). Die Interviews wurden vollständig transkribiert, wobei sämtliche Personen- und Ortsnamen anonymisiert wurden. Diese Materialien werden in der Forschungsgruppe in Anlehnung an das Verfahren der Grounded Theory ausgewertet (Strauss 1998). Bei der DatenanaIyse dienen sozialtheoretische Überlegungen zu einem relationalen Verständnis von Agency und Vulnerabilität (Burkitt 2015) sowie methodologische Überlegungen zu subjektiven Konzeptionen von Agency (Helfferich 2020) als sensibilisierende Zugänge und Konzepte.

\section{ANKOMMEN AUS SICHT DER JUGENDLI- CHEN}

Die im Rahmen unserer Untersuchung bisher geführten Interviews deuten auf ganz unterschiedliche Erfahrungen in Bezug auf den Herkunftskontext, die Flucht, die Ankunft und das 
bisherige Leben in der Schweiz hin. Vor diesem Hintergrund wurden für diesen Beitrag zwei Einzelfälle ausgewählt, bei denen sich unterschiedliche Sichtweisen auf das Ankommen in der Schweiz zeigen. D. h. sie unterscheiden sich insbesondere in ihrer Wahrnehmungsweise der institutionellen als auch strukturellen Bedingungen ihres Ankommens in der Schweiz, die sie entweder als begrenzenden oder grenzenlosen Möglichkeitsraum für sich erleben. Um zu verstehen, was Vulnerabilität und Agency für die Jugendlichen in den jeweiligen Kontexten und sozialen Beziehungen wie auch unter unterschiedlichen sozialen Bedingungen bedeuten, erscheint es lohnenswert, ihre Erzählungen zu den unterschiedlichen biographischen Lebensphasen ihres Migrationsprozesses zu berücksichtigen. Im Rahmen einer ersten Falldarstellung wird auf Aras eingegangen, der vor der Fluchtmigration familiale Fürsorge erfahren hat und sich nun als selbständige, erwachsene Person positioniert (3.1). Anschliessend werden wir im Rahmen einer zweiten Falldarstellung auf Ahmend eingehen (3.2), der dadurch aufgefallen ist, dass er Diskontinuitäts- und Übergangserfahrungen als Normalzustand beschreibt. Die Erzählungen der Jugendlichen als biografische Selbstthematisie- rungen sind hierbei als retrospektive, auf die gegenwärtige Situation und Zukunft erfolgende Sinn- und Bedeutungskonstruktionen zu verstehen.

\subsection{Aras - „von Null anfangen und dann geht's weiter"}

Geboren und aufgewachsen ist Aras in Syrien und 2015 ist er über die europäischen Fluchtmigrationsrouten in die Schweiz gekommen. Seine Eltern leben zum Zeitpunkt des Interviews noch immer in Syrien, während zwei seiner Schwestern in anderen europäischen Ländern leben. Über seine Reiseroute und auf der Flucht gemachte Erfahrungen spricht Aras im Interview nicht. Zum Zeitpunkt des Interviews ist Aras 17 Jahre alt, verfügt über den vorläufig aufgenommenen Asylstatus „F“, ist gerade in ein Ein-Zimmer-Apartment in Grossstadt 1 gezogen, besucht weiterhin das letzte Jahr der Sekundarschule in Stadt 1, bevor er im September 2019 eine Ausbildung in einem bautechnischen Bereich beginnen wird (vgl. Abb. 1). ${ }^{4}$ Sein Onkel und dessen Familie leben in unmittelbarer Nähe der eigenen Wohnung.

Abbildung 1: Lebensstationen Aras - gemäss Interviewerzählung

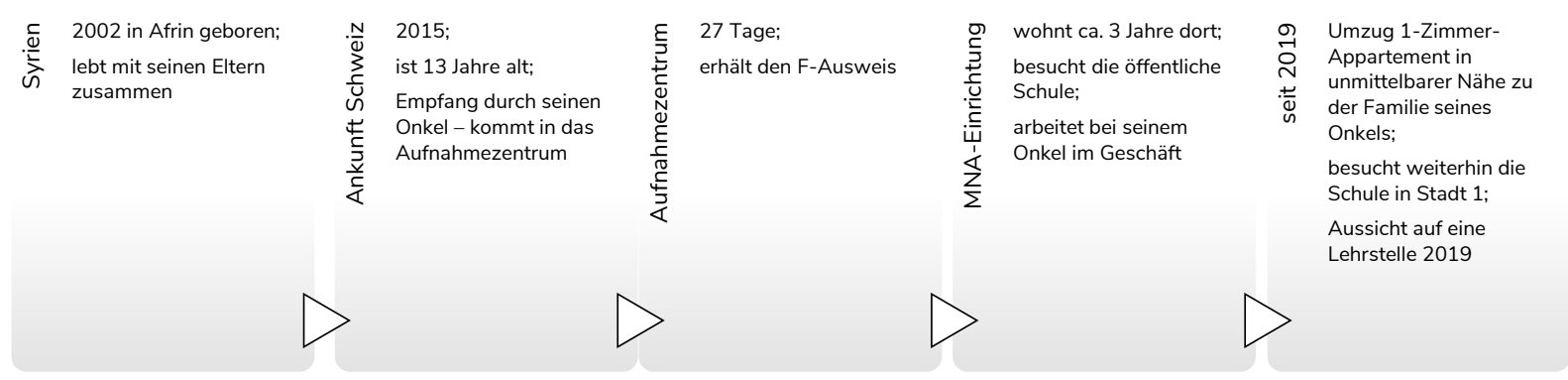

Quelle: eigene Darstellung

\subsubsection{Herkunftskontext}

Aras gibt an verschiedenen Stellen Einblicke in sein familiäres Beziehungsgefüge und die Bedingungen seines Aufwachsens vor der Flucht, wie es in der folgenden Erzählung zum Ausdruck kommt:
"Ja als ich noch in Syrien war, waren meine Eltern für mich zuständig oder sie haben mir alles gemacht und alles (.) es waren einfach (.) für mich da oder sie haben mir alles gemacht einfach gekocht und auch was wie immer oder Sachen gekauft (.) also ich musste mir einfach keine Sorgen machen [...]." (Z.61)

\footnotetext{
${ }^{4}$ Mit dem Asylstatus „F“ werden Personen als Flüchtling anerkannt, sind allerdings nach nationalem Recht von Asyl ausgeschlossen. Sie werden in der Schweiz vorläufig aufgenommen. Der Ausweis wird jeweils für ein Jahr ausgestellt und kann verlängert werden.
} 
Später kommt er erneut auf seinen Herkunftskontext zu sprechen:

„[U]nd in Syrien (.) hatte ich nie so eine fremde Person getroffen (.) es waren [...] einfach alle (.) die meine Sprache gesprochen haben (und dann) hatte ich auf einmal (.) viele Leute die (.) keine Ahnung andere Sprache sprechen oder (.) andere (.) Nationalitäten haben und [...] vor allem so (.) dunkelhäutige Menschen und das habe ich noch nie im Leben gesehen @.@ (I: Mhm.) erst in der Schweiz (.) ja (.).“ (Z. 953-960)

Aras beschreibt das Aufwachsen in einem von Fürsorge geprägten familialen Umfeld, in dem er eine sorgenfreie Kindheit erlebt hat. Zudem skizziert er den Kontext seines Aufwachsens als einen, in dem nur die vertraute Sprache und vertraut aussehende Menschen präsent waren. Erst nach seiner Flucht macht er Fremdheitserfahrungen, wie die mit anderen Sprachen verbundene Möglichkeit von Verständigungsproblemen oder das Zusammenleben mit Menschen anderer natio-ethno-kultureller Zugehörigkeiten. Die Entbehrung der Eltern sowie die Fremdheitserfahrungen in der Schweiz gehen mit der Erfahrung von Neuem und Irritationen für Aras einher und fordern ihn auf, seine bisherigen Erfahrungen weiterzuentwickeln.

An anderer Stelle im Interview kommt Aras nochmals auf die Abwesenheit seiner Eltern in der Schweiz zurück. Er erzählt, dass er seine Eltern aufgefordert habe, in die Schweiz zu kommen, diese sich allerdings dagegen ausgesprochen haben. Auf die Rückfrage der Interviewerin, wie dies für inn sei, reagiert er mit einer Normalisierungstaktik und einem generationalen Vergleich:

„[E]s hat Vorteile und Nachteile (.) in die Schweiz kommen oder (.) dann sie sind ja nicht so jung wie ich oder (I: Ja.) sie sind ältere Leute oder (.) [...], ich bin sicher dass sie so Schwierigkeiten haben werden oder (.) beim [...] integrieren und so und die Sprache (eben) so Deutsch und (.) [...], weil dort sie sich keine Ahnung sich einfach frei fühlen oder (.) es ist schon nicht sicher oder (.) s ist schon Krieg dort oder (I: Mhm.) (.) aber sie sind halt dort geboren und sie haben alles im Griff oder (.) sie dürfen Auto fahren sie dürfen (3) alles machen was sie wollen oder (.) und wenn sie in die
Schweiz kommen oder (.) da sind sie halt Flüchtlinge oder da müssen sie (.) in ein Heim gehen wo sie nicht wollen oder (.) da würden sie sich schlecht fühlen oder (.) mit fünfzig Jahren in ein Heim gehen oder (.) und [...] müsst einfach essen und schlafen huh das wollen sie irgendwie nicht oder (I: Mhm.) das ist einfach ähm (.) unangenehm." (Z. 669-693)

Eindrücklich beschreibt Aras in dieser Sequenz den mit dem Fluchtmigrationsprozess verbundenen Verlust an Möglichkeitsbedingungen von Autonomie sowie damit einhergehenden Verlusterfahrungen des Gewohnten: Verloren wird die eigene Sprache und die uneingeschränkte Bewegungsmöglichkeit. Mit der Ankunft in der Schweiz werden seine Eltern "Flüchtlinge" und geben damit gewissermassen das Recht auf ein selbstbestimmtes Leben ab: „Einfach essen und schlafen" bedeutet innerhalb der vorgegebenen Strukturen zu funktionieren, sich unterzuordnen und abzuwarten. Aras skizziert also in Bezug auf den Aufnahmekontext situative Vulnerabilitätsbedingungen, die für inn als junger Mensch noch zumutbar sind - für seine Eltern jedoch unzumutbar erscheinen; Leben unter Bedingungen des Krieges erscheint hier als das geringere Übel. Die mit der Erzählung verbundene subjektive Bewertung der Situation deutet an, dass Aras sich unter prekären Bedingungen der Asylpolitik Handlungsmöglichkeiten erarbeitet hat, und lassen Agency als dynamisches Durchhalten erscheinen, das aber nicht für jeden zumutbar ist.

\subsubsection{Ankunft im Erstaufnahmezentrum: ein be- grenzter Möglichkeitsraum}

Die Beurteilung der mit Fremdbestimmung verbundenen Migrationserfahrungen sowie der Herausforderungen in Bezug auf einen als anspruchsvoll erlebten Integrationsprozess im Aufnahmekontext für seine Eltern ist verknüpft mit seinen eigenen Erfahrungen der Ankunft in der Schweiz. Nachdem er mit dem Zug am Bahnhof angekommen ist und dort von seinem Onkel in Empfang genommen wurde, hat dieser inn in ein Erstaufnahmezentrum gebracht, welches für ihn mit Angst und Überforderung verbunden ist:

„Das Heim war einfach voller Leute oder und da war ich (.) und (.) ( ) hatte gesagt du musst jetzt reingehen (.) so, wie ein Stall (.) und jetzt hier 
müssen wie jetzt reingehn und die Türe zu oder (.) und es gibt niemand mit dem man reden kann oder und dem man Fragen stellen kann oder (.) keine Übersetzer und niemand fragt dich (.) und man kann einfach keine Fragen stellen wieso ich jetzt da bin oder was passieren kann oder (.) einfach so oder (.) und dann musst du einfach reingehen und darfst nicht mehr raus gehen (.) du musst einfach essen und warten bis jemand dich holt oder und interviewt und so (.) dann war ich dort und hab ich so ein komisches Gefühl gehabt (.) mir war immer schlecht ich habe nicht gewusst (.) he das ist so komisch wieso bin ich da ich verschwinde hier und ich hab so oft geweint ich habe mich so einsam gefühlt (.) he ich will weg von hier." (Z. 132-148)

Sehr anschaulich schildert Aras seine Eindrücke von dem für ihn unverständlichen Ankunftskontext, der für inn mit Gefühlen der Orientierungslosigkeit und des Eingesperrtseins verbunden ist. Dass er sich schlecht fühlt, sich nicht orientieren kann, weinen muss und einsam ist, mögen als spezifische emotionale Verfasstheiten von Aras Vulnerabilität in der Situation des Ankommens zeugen und auf einen für ihn begrenzten Möglichkeitsraum verweisen.

Deutlich merkt man seiner Sprechweise die für inn immer noch aktuelle Erschütterung angesichts dieser existentiellen Erfahrung an, der er sich ausgesetzt fühlt und sich dieser gegenüber auch nicht verwehren kann. Er erzählt an anderer Stelle: "[l]n diesem Heim war ich 27 Tage und es war ziemlich anstrengend." Sowohl die hier konkret erinnerte Zeitlichkeit als auch die verbalisierten Gefühle von Aras verweisen darauf, dass es sich bei der Ankunft um ein für ihn einschneidendes Erlebnis handelt. In der Schweiz erfährt er nicht die bisher erlebte Fürsorge; er ist auf sich alleine zurückgeworfen und leidet unter Ungewissheit, Einsamkeit und Angst sowie dem fehlenden Mitbestimmungsrecht hinsichtlich des Verlaufs seiner Unterbringung.

\subsubsection{Von der MNA-Einrichtung in die eigene Wohnung}

Aras lebt jahrelang in einer MNA-Einrichtung und bezieht von dort aus, kurze Zeit vor dem In- terview, ein eigenes 1-Zimmer-Appartment. Seinen Alltag in der Schweiz erlebt er durchaus ambivalent. So erzählt er zunächst:

„[S]o mein Alltag in der Einrichtung war einfach immer so stressig weiss nicht wieso (.) mir war immer stressig (.) also ich muss immer so früh aufstehen (.) und dann in die Schule gehn [...] und am Nachmittag ging ich wieder in die Schule (.) und am Abend halb sechs Hausaufgabenhilfe (.) und da haben sie mich unterstützt bei den Aufgaben ((husten)) und schlussendlich musst ich noch kochen, essen, und duschen und dann schlafen (l: Mhm.) ja (sagen wir) immer so gleich (.) immer so stressig." (Z. 179-191)

In dieser atemlos wirkenden Aufzählung seiner alltäglichen Verrichtungen fällt die häufige Verwendung des Attributes "stressig“ auf, wobei die „immer so gleich(en)“ Tage als Abfolge von Verpflichtungen geschildert werden. Den dann erfolgenden Umzug in die eigene Wohnung, verbindet Aras mit einem Statuswechsel. Er bringt dies wie folgt zur Sprache:

„Ja (.) ich fühl mich so eher selbständig (I: Mhm.) @.@ nicht mehr so (.)@wie ein Kind@ @.@ (.)ja [...]. Ja eigentlich Selbständigkeit war schon immer auch im Birkenhof oder (.) aber da ich jetzt alleine wohne ist noch mehr selbständig (oder) da ich so allein in ein Zimmer bin oder und niemand (.) ähm niemand mich betreut oder niemand jetzt im Haus für mich zuständig ist oder (dann bin) ich einfach selbständig (.) ich muss einfach (alles) (.) selbständig entscheiden (organisieren) oder (I: Mhm.) wie ein erwachsene Person." (Z. 40-53)

In dieser Sequenz grenzt Aras sich vom „KindSein" ab und positioniert sich als "erwachsene Person": Als diese muss er seinen Alltag nun gänzlich selbst gestalten. Insbesondere der Übergang von der Heimunterbringung in die eigene Wohnung ist für inn mit einem Zuwachs an Selbstständigkeit verbunden. Der Zuwachs an alltäglicher Autonomie zeigt sich bei Aras jedoch auch als eine Form der Überforderung, nun eben alles selbstständig - und für sich selbst verantwortlich - entscheiden zu müssen. Inzwischen, so Aras weiter, habe er sich daran gewöhnt, all diese Pflichten selbstständig zu erledigen, auch wenn er es als mühsam empfinde, all dies alleine 
bewältigen zu müssen. Gleichzeitig ist Aras auf soziale Unterstützung angewiesen. Denn die Wohnung, die er von der Asylorganisation zugewiesen bekommt und beziehen darf, ist, so Aras, „leer, und ich habe ja keine Küche“. Sofern er nicht über die notwendige materielle Ausstattung verfügt, lässt sich dies als eine strukturell verhinderte Agency lesen, die Aras sehr deutlich im Interview kritisiert und die inn in seinen Möglichkeitsbedingungen der selbstverantwortlichen Gestaltung des Alltags einschränkt. Hierbei bleibt seine Verwandtschaft - und insbesondere sein Onkel - auch weiterhin die für inn signifikante Gruppe von (Bezugs-)Personen, die ihm während der Zeit Unterstützung gewährt und Kontinuität sicherstellt (Göbel et al. 2020). So erzählt Aras: „[D]ann kochen sie für mich, oder weil sie da in der Nähe sind, da kann ich jeden Tag hingehen und dort essen und dann halt schlafen und Hausaufgaben machen". Aus einer eigenen emotional wertschätzenden Perspektive kann Aras in dieser Konstellation sozialer Unterstützung für sich Handlungsfähigkeit herstellen, seinen Alltag selbstständig gestalten und sich auf die Schule konzentrieren.

\subsubsection{Das Asylverfahren: Überzeugungsarbeit leisten und Kampfgeist entwickeln}

Die oben skizzierte Verbindung aus zeitlich ausgedehntem Erleben unangenehmer Verpflichtungen, die Aras in der Schweiz zu absolvieren hat, zeigt sich ausserdem in der Erzählung über die Anhörung im Rahmen seines Asylverfahrens. Er berichtet in diesem Zusammenhang: „[S]ie haben die Fragen immer wiederholt, also das hat stundenlang gedauert" (Z. 652). Hierbei ist das Asylverfahren für inn mit der Anforderung verbunden, eine Überzeugungsarbeit zu leisten, um in der Schweiz bleiben zu können und eine "gute Aufenthaltsgenehmigung" zu erhalten. Aras Asylstatus „F“ gehört nicht dazu: Dieser schränkt ihn in seinen Möglichkeitsbedingungen ein und ist mit spezifischen Anforderungen, wie z. B. der erfolgreichen Lehrstellensuche, verbunden. Inwiefern es sich hierbei um eine existentielle Erfahrung der totalen Fremdbestimmung handelt, wird dann deutlich, wenn Aras wie folgt beschreibt: „[W]enn [der Richter] überzeugt ist, dass du wirklich wegen dem Krieg geflüchtet bist, dann bekommst du einen guten Aufenthalt wie im B-Politik, wenn der nicht glaubt, bekommst du einen F normal, wie ich". Es sind die Fremdheitsgefühle, die Erfahrungen der Ablehnung, die existentielle Ohnmacht gegenüber strukturellen Bedingungen sowie Diskriminierungserfahrungen durch das Asylsystem, die auf kontextspezifische und situative Vulnerabilitätserfahrungen verweisen. Gleichzeitig erarbeitet sich Aras einen Kampfgeist, der sich affirmativ zu den sozialen Verhältnissen der Prekarität und Ungerechtigkeit verhält:

„[D]as macht keinen Sinn wenn wir darüber reden oder (.) [...] den ganzen Tag (schimpfen) oder (.) (weinen) das macht keinen Sinn @.@ das ist halt Politik (.) wir müssen einfach (.) darauf verzichten und halt [...] kämpfen oder (.)." (Z. 840845)

Auf Rückfrage der Interviewerin, was er denn mit kämpfen meine, fährt er wie folgt fort:

„Ja halt [...] sich weiter entwickeln oder (.) einfach weiter in die Schule gehn und versuchen (.) [...], einfach selber weiter zu kommen oder (.) etwas mit der Schule zu machen oder (.) wenn du die Sprache kannst wenn du in die Schule gehst oder (.) kommst du auch etwas besser (zurecht) (.) halt so ja." (Z. 849-853)

Obwohl Aras die strukturellen Rahmenbedingungen als Zwang empfindet, erscheint es für inn als sinnlos, sich den selektiven und regulativen Logiken des Asylsystems zu widersetzen. Die von ihm eingenommene kämpferische Grundhaltung ist damit auf die Normalisierung seines Ankommens wie auch Disziplinierung seiner selbst gerichtet. In Bezug auf die Herstellung seiner Handlungsfähigkeit in dem Aufnahmekontext Schweiz müsse er sich in Verzicht üben und gleichzeitig eine anpassungsfähige Haltung gegenüber dem Asylsystem und den damit verbundenen Anforderungen der Aufnahmegesellschaft einnehmen. Gefühle der erlebten sozialen Ungerechtigkeiten, wie sie sich in Wut oder Weinen ausdrücken, gilt es hierbei zu unterdrücken (Magyar-Haas 2017, 49). Vielmehr ist Konzentration und Durchhaltevermögen in Bezug auf die an ihn gestellten Anforderungen der Aufnahmegesellschaft gefordert: Nur die erfolgreiche (Aus|Bildung, der kompetente Erwerb der hegemonialen Sprache und das Entsprechen neoliberaler 
Subjektanforderungen des „Weiterkommens“ ermöglichen ein „erfolgreiches“ Absolvieren des Asylverfahrens und eine auf die Zukunft ausgerichtete Bleibeperspektive in der Schweiz.

Mit Blick auf die Veränderungen, Brüche und Diskontinuitäten innerhalb des Fluchtmigrationsund damit verbundenen Ankommensprozesses von Aras legt die Fallanalyse nahe, die Diskontinuitätserfahrungen nicht ausschliesslich auf eine kategoriale Dimension des Vulnerabel-Seins zu reduzieren (Clark 2007). Aras übt sich in Verzicht und nimmt damit Vulnerabilitätserfahrungen, wie die Abwesenheit seiner Eltern im Hier-und-Jetzt, für sich an. Er entwickelt eine anpassungsfähige Haltung in Bezug auf die soziale Integration im Aufnahmekontext und damit in das Asyl- und Bildungssystem, um den situativen Bedingungen nicht mehr machtlos ausgeliefert zu sein. Es sind die situativen Vulnerabilitätsbedingungen (Mackenzie et al. 2013), die mit der Herstellung als auch Erarbeitung einer kontextspezifischen Agency einhergehen, die eben bedeuten kann, einen „Kampfgeist“ zu entwickeln, denn so Aras im Interview: „weiter geht's“.

Während Aras geschilderte Erfahrung familialer Fürsorge und Bedingungen des Aufwachsens vor dem Migrationsprozess mit einem eurozentrisch dominierenden bürgerlichen Konzept einer umfassend betreuten Kindheit in Einklang gebracht werden können, verweisen die Erzählungen von Ahmend auf einen ganz anderen Hintergrund.

\subsection{Ahmend - „Ich bin ja immer neu für mich ist einfach normal.“}

Ahmend wurde in Somalia geboren, bevor seine Eltern nach Äthiopien migrierten. Nach dem Tod seiner Eltern als auch seines Onkels verbringt Ahmend seine Kindheit auf der Strasse. Ca. 2016 ist er über Italien in die Schweiz gekommen. Über seine Erfahrungen auf der Flucht spricht auch er im Interview nicht. In der Schweiz angekommen, wird er im Unterschied zu Aras nach dem Erstaufnahmezentrum in einer Pflegefamilie platziert. Zum Zeitpunkt des Interviews ist Ahmend 15 Jahre alt und lebt in seiner zweiten Pflegefamilie mit zwei weiteren Kindern. Er besucht die öffentliche Schule, absolviert verschiedene Schnupperlehren, hat einen Nebenjob in einer Metzgerei und verfügt - wie Aras - über den Aufenthaltsstatus „F“. Zudem stellt das Fussballspielen eine Kontinuität in seinem Leben dar. Zum aktuellen Zeitpunkt spielt er in einer U16-Mannschaft und träumt von einer Karriere als Profifussballer. Seine Stationen lassen sich wie folgt darstellen:

Abbildung 2: Lebensstationen Ahmend - gemäss Interviewerzählung

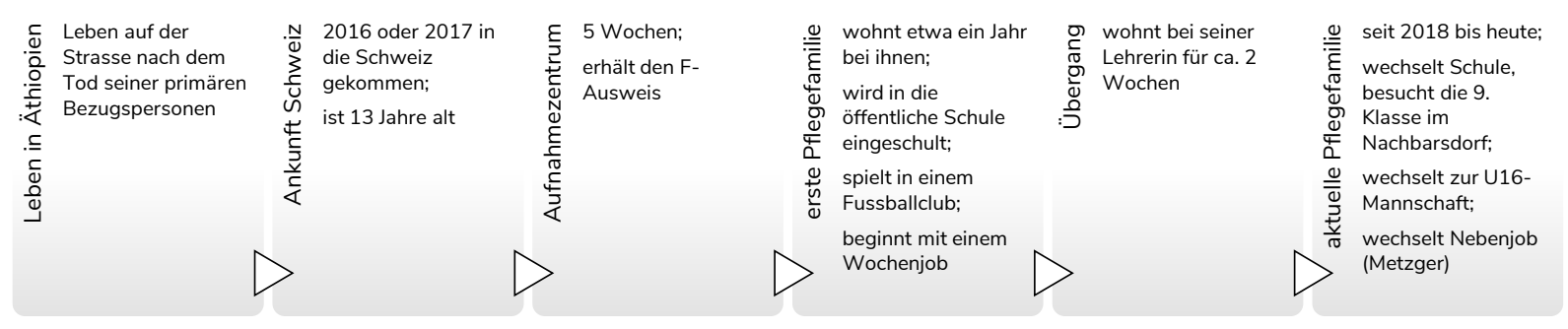

Quelle: eigene Darstellung in Anlehnung an Niggli-Gamper $(2019,86)$

\subsubsection{Herkunftskontext}

In Bezug auf seine Erfahrungen im Herkunftskontext berichtet Ahmend wie folgt:

„Meine Eltern sind, keine Ahnung zwischen 6 oder 8 bin ich gewesen, gestorben wegen so eine nicht gerade Krieg aber wegen einfach ein paar Leute [...], sie haben so meine Eltern getötet (.) und nachher bin ich mit - mein Onkel hat mich in die Hauptstadt von - zweite Hauptstadt von Äthiopien mitgenommen (.) er hat nachher nach einem Jahr so eine Krankheit gehabt im Bein und so und ist er auch gestorben und nachher habe ich müssen auf der Strasse leben dort - ja dort habe ich auch gute Kollegen gehabt, habe ich müssen einfach Schuhe putzen oder ein Auto putzen oder irgendetwas gemacht für das ich Geld habe für das Essen oder irgendetwas [...] lch habe auf der Strasse gelebt [...], ich habe nicht so 
leben können wie jetzt (3) habe Arbeiten müssen, Mittagessen suchen alles suchen (3) (.) nicht so können duschen oder nicht Haare schneiden können alles ist einfach schwieriger gewesen. Ich habe nicht können Kleider leisten." (Z. 14511461)

In dieser Passage wird deutlich, inwiefern das Leben von Ahmend bereits während der Kindheit durch den Verlust wichtiger Bezugspersonen und für inn Sorgenden, durch die Notwendigkeit der Binnenmigration, durch Obdachlosigkeit sowie die Notwendigkeit, durch Arbeit den eigenen Lebensunterhalten zu sichern, gekennzeichnet war. Auf Grund der prekären und vulnerablen Lebensverhältnisse ist Ahmend dazu aufgefordert, sich Selbstständigkeit zu erarbeiten, die „pragmatisch auf die Bewältigung der täglichen Erfordernisse zielt“ (Liebel 2017, 43) wie auf das Erreichen einer gewissen Form sozialer Verlässlichkeit (Payne 2012). Letzteres zeigt sich darin, dass Ahmend sich soziale Netzwerke schafft und davon spricht, dass er "gute Kollegen“ gehabt habe, die sich gegenseitig unterstützten aber auch miteinander Fussball spielten:

"[l]ch bin eigentlich immer froh gewesen ja ich habe ja keine Ahnung gehabt wie es hier läuft [...], wenn ich gewusst hätte wäre es mir dort einfach wahrscheinlich schlecht gegangen aber wenn ich so einfach für mich ist einfach mega gut gewesen dort [...], es hat alles so Spass gemacht mit Kollegen ein Spielen einfach ja [...], aber wenn man es nicht besser leben kann muss man ja einfach froh sein dort [...], weil du kannst ja nichts dafür machen." (Z. 1448-1454)

Aus einer die eigene Situation evaluativ bewertenden Perspektive nimmt Ahmend die Beschreibungen eines durch Entbehrung und Mühsal gekennzeichneten Lebens auf der Strasse als solches erst nachträglich wahr. Gleichzeitig vollzieht er eine Normalisierung jener Bedingungen des Aufwachsens. Die damalige Zufriedenheit mit dem Leben begründet er mit fehlendem verfügbarem Wissen über die ihm zur Verfügung stehenden Möglichkeitsbedingungen seiner gegenwärtigen Lebenssituation - wie bspw. Zugang zu Bildung. Doch trotz der prekären Lebensbedingungen sei er immer „froh" gewesen.
3.2.2 Ankunft im Erstaufnahmezentrum: ein grenzenloser Möglichkeitsraum

In Bezug auf die Ankunft in der Schweiz erzählt er wie folgt:

"Ich habe es mega cool gefunden dort so viele Leute (.) kannst einfach so leben also wir haben bis um 5 Uhr dürfen draussen sein (2) und nachher haben wir pro Woche 7 Franken bekommen ist mega cool gewesen (3) haben wir können draussen herumchillen Frühstück alle zusammen - können essen mit 200 Personen oder 300 ein super feines Frühstück auch ein feines Mittagessen am Abend Filmabend immer das ist cool gewesen ich vermisse sogar (.) manchmal [...] und Filmabend haben wir eben nicht gekonnt weil ich bin 12 Jahre gewesen ich habe nicht dürfen Filme sind manchmal über 18 und so gewesen [...] und nachher haben wir uns versteckt sind wir nachher immer rüber gegangen (.) nachher haben wir die Nacht dort geschaut bis um 12 Uhr Filme oder 11 Uhr und nachher sind wir eben zurück gekommen, schlafen gegangen (3) es hat mega Spass gemacht dort (2) ja (3) es hat einfach so viel Leute gehabt weisst du (9) ich habe es auch gerne wenn es mega viele Leute hat dann kannst du mit jedem chillen einfach." (Z. 925942)

Während Aras seine Ankunftserfahrungen in der Schweiz als einen deutlichen Bruch mit seinem bisherigen Leben präsentiert, zeigt sich in der Erzählung bei Ahmend der Bruch mit seinen bisherigen Erfahrungen auf konträre Art und Weise. Das Erstaufnahmezentrum symbolisiert einen grenzenlosen Möglichkeitsraum, statt einen Ort strukturell bedingter Abhängigkeiten und Vulnerabilitätserfahrungen. Geschildert wird ein Raum, der es Ahmend ermöglicht, mit vielen Menschen zusammenzuleben, materielle Fürsorge zu erfahren und sich zu erholen. Während er in Bezug auf seinen Alltag in Äthiopien eine fürsorglose Autonomie beschreibt, macht er nun materielle Fürsorgeerfahrungen durch andere und ist gleichzeitig in seiner gewohnten Bewegungsfreiheit und sozialen Beziehungspflege nicht eingeschränkt. Zugleich relativiert er diesen grenzenlosen Möglichkeitsraum an anderer Stelle, wenn er rückblickend sagt: „nur eine Woche dorthin zurück, nicht länger". 
Eine unterschiedliche Erlebensweise zu Aras wird auch mit Blick auf das Asylverfahren deutlich. Ahmend beschreibt dieses als eine Art Parcours, bestehend aus zwei Anhörungen, den er gemäss seinen Schilderungen besonders schnell absolvierte: „Ich bin der schnellste gewesen, niemand hat es so geschafft gehabt [...], ich habe glaube ich dort einen Rekord gemacht" (Z. 909). Diese Zeit der Anhörungen im Rahmen des Asylverfahrens scheint hier wie im Flug zu vergehen und auch noch als Bestärkung des eigenen Selbstwertgefühls erlebt zu werden. Für Ahmend symbolisiert das erfolgreiche Absolvieren des Asylverfahrens gleichzeitig den Erhalt eines legitimen Status in der Schweiz. Erst viel später in seinem weiteren Werdegang erlebt er Einschränkungen auf Grund des inm verliehenen Aufenthaltstatus „F“, denn seinen Traum Fussballprofi zu werden und in einen besseren Fussballclub zu wechseln, kann er mit diesem Status nicht verwirklichen.

3.2.3 Platzierung in einer Pflegefamilie: Positionierung als kleines Kind und situativer Autonomieverlust

Danach wird Ahmend in seiner ersten Pflegefamilie platziert, in der er ungefähr ein Jahr lebt. Seine erste Begegnung mit der Pflegefamilie beschreibt er als „komisch“. Denn er wird mit Familien- und Vatervorstellungen konfrontiert, die nicht seinen eigenen entsprechen und zu Verunsicherungen führen. So ist es für Ahmend irritierend, dass der für inn zukünftig sorgende Pflegevater erst 28 Jahre alt ist und "schon“ drei Kinder hat, und er erzählt: „[N]achher habe ich gedacht, er ist nicht Mann, er ist nicht Vater, er ist einfach normal, einer der 18 ist" (Z. 1074-1082). Während er sich in seiner aktuellen Pflegefamilie unterstützt und wohl fühlt, ist in der ersten Pflegefamilie also alles ,anders“ (Z. 205) gewesen, er führt auf die Aufforderung „erzähl mal“ seitens der Interviewerin aus:

"[D]ort ist einfach sie haben mich einfach so wie kleines Kind behandelt sie haben (.) ich habe immer schon so am 9 Uhr zuhause sein müssen oder am 8 Uhr (.) sie haben ich habe pro Tag ungefähr eine Stunde dü- dürfen Handy brauche Internet (7) ja (7) [...]. Sie hat kleine Kinder gehabt und nachher dort ist einfach wie Kinder gegangen hast ja nichts Spezielles machen können [...] und nachher habe ich müssen alles mitmachen was kleine Kinder machen." (Z. 205-216)

In dieser Sequenz beschreibt Ahmend nicht nur eine negative Aufzählung an alltäglichen Restriktionen, sondern auch eine Gleichschaltung mit den Kindern aus der Pflegefamilie. Indem er "wie ein kleines Kind behandelt" wurde, macht er die dort erfahrene Fremdbestimmung deutlich und weist implizit daraufhin, dass er sich als Person nicht ernst genommen fühlte. Diese Formulierung weist auf eine Differenzerfahrung von Ahmend hin: Er ist als Jugendlicher mit Migrationserfahrung anders und positioniert sich als nicht-zugehörig. Die Differenzerfahrung als Kind positioniert zu werden, drückt sich für Ahmend darin aus, dass sich sein Tagesablauf als auch seine Freizeitaktivitäten an denen der jüngeren Kinder der Pflegefamilie orientieren müssen und er sich unzulässig bevormundet fühlt. So erzählt er an anderer Stelle, dass er keine Fussballspiele schauen dürfe und sein Medienkonsum auf eine Minimaldauer pro Tag festgelegt wird. Damit wird Ahmend in seinen Wünschen und Bedürfnissen eingeschränkt: Denn während der Fussball immer eine Konstante in seinem Leben darstellte, die ihm Zugang zu seinen Träumen und Zukunftsvorstellungen als auch seinen Peers ermöglichte, ist der unbeschränkte Zugang zu einem funktionierenden WLAN für inn insofern wichtig, als er darüber seine digitalen sozialen Netzwerke pflegt. Seine Differenzerfahrung als Person mit Migrationserfahrung beschreibt er an anderer Stelle, wenn er sich implizit selbst als „Ausländer" positioniert:

"[l]ch habe damals nicht einmal Deutsch gekonnt (I: Mhm.) ja ich habe niemanden gekannt gehabt [...] dort ich habe auch nicht Somalier oder so gekannt weisst du es ist so ein Dorf wo kein Bahnhof hat (.) du kannst nirgendwo hin gehen und dort hat es keine Ausländer fast." (Z. 220-228)

Sowohl die sozialräumliche Isolation als auch die nicht vorhandenen Sprachkenntnisse gehen für Ahmend mit zu bewältigenden Herausforderungen einher. Mit der Platzierung in der ersten Pflegefamilie verliert er den Kontakt zu seinen bisher aufgebauten sozialen Beziehungen in dem Erstaufnahmezentrum und zu seinen virtuellen sozialen Netzwerken, ist in seiner Mobilität eingeschränkt und verfügt über keine Personen 
in seinem nahen Umfeld, mit denen er seine vertraute Sprache sprechen kann. Die mangelnden Deutschkenntnisse führen zu dieser Zeit bei Ahmend dazu, dass er innerhalb der Familie nicht alles versteht und sich dadurch nicht angemessen beteiligen und einbringen kann.

\subsubsection{Abbruch des Pflegefamilienverhältnisses}

In der Folge kommt es zum Abbruch des ersten Pflegefamilienverhältnisses. Ahmend erzählt zum einen von Bestrafungen, die sich für ihn in fehlender materieller Fürsorge ausdrücken, wenn inm das Essen verweigert wird, zum anderen von körperlichen Gewalterfahrungen, die das Ende des Pflegefamilienverhältnisses einläuten:

„[N]achher ist der Mann am Abend gekommen und hat er mich so wegstossen gibt mir alles Geld ich habe gesagt ich gebe dir nicht und nachher bin ich einfach weggegangen." (Z. 269-271)

Es sind die von ihm beschriebenen Bedingungen asymmetrischer und machtvoller Sorgebeziehungen, die auf situative Vulnerabilitätserfahrungen und damit Entmachtung seiner für ihn bislang tonangebenden ganz eigenen Vorstellungen und Praktiken der Lebensführungsweisen hindeuten. Der reglementierte Alltag wie auch die Positionierung als Kind scheinen im Widerspruch zu Ahmends gewohnten und vertrauten Lebensführungsweisen zu stehen, in denen er in erster Linie für sich selbst verantwortlich gewesen ist, und führen damit zu einer Einschränkung seiner gewohnten Agency.

Ahmend beschreibt zudem mangelnde Partizipationsmöglichkeiten in Bezug auf den Platzierungsprozess als auch die asylpolitischen Strukturen, die keine klaren nachvollziehbaren Regeln vorgeben. Die Platzierung von Ahmend in einer Pflegefamilie erscheint hier als infantilisierende Bevormundung, sofern er gerade nicht Teil des Entscheidungsprozesses ist.

\subsubsection{Von der unzulässigen Bevormundung zur Selbstermächtigung}

Vor diesem Hintergrund lässt sich die oben aufgeführte Passage, in der Ahmend berichtet, dass er auf Grund der ihn vulnerabilisierenden Bedingungen in der Pflegefamilie „einfach gegangen“ sei, als eine Selbstermächtigung deuten. Denn bei der dann erfolgenden Umplatzierung ergreift er selbst die Initiative. Hierfür greift er auf seine sozialen Beziehungs- und Unterstützungsnetzwerke zurück, die er sich bislang in der Schweiz aufgebaut hat, um der Situation, mit der er unzufrieden ist, Abhilfe zu schaffen. Er erzählt, dass er zunächst bei einem Kollegen übernachtet habe, bevor er bei seiner damaligen Lehrerin eine Zwischenlösung gefunden hat. Von dort aus habe er dann Kontakt mit seiner für ihn zuständigen Sozialpädagogin aufgenommen, um mitzuteilen, dass er nicht länger dort bleibe. Während in der ersten Familie kein Prozess des Kennenlernens stattfand, wird Ahmend nun nicht nur an dem Prozess beteiligt, sondern er bindet sich selbst aktiv ein: Er besteht auf einer "Schnupperphase“ und dem Kriterium, dass er nicht mehr mit kleinen Kindern zusammenleben möchte.

Die Anfangsphase und der damit verbundene Prozess des (erneuten) Ankommens in einer anderen Pflegefamilie erlebt Ahmend dann nicht mehr als eine radikale Fremdheitserfahrung, wenn er erzählt: „[F]ür mich ist nachher alles normal gewesen, weil ich bin ja fast zwei Jahre in der Schweiz gewesen". Der hier von Ahmend angesprochene Normalisierungsprozess deutet auf die Bewältigung von begrenzenden und sich gleichzeitig eröffnenden Möglichkeitsräumen hin. Zum einen kann er sich nun in der neu erlernten Sprache mit den Familienmitgliedern verständigen; zum anderen beschreibt Ahmend einen Zuwachs an Mitbestimmungsrechten:

„[D]ie [Pflegefamilie 1] haben das einfach so gemacht und nachher habe ich einfach immer müssen mitgehen (l: Mhm.) (.) aber hier ist meine Entscheidung ich kann sagen ob ich mitkommen möchte." (Z. 1190-1192)

Ahmend betont, dass er nun in seinen Entscheidungen respektiert wird und auch seine Bedürfnisse selbstbestimmt und uneingeschränkt artikulieren kann. Zudem berichtet er, wie die aktuelle Pflegefamilie inn bei dem Wechsel in eine neue Schule und einen neuen Fussballclub sowie in der Akquise eines Nebenjobs unterstützt. Damit verbunden ist die Wiederaufnahme seiner sozialen Kontakte, die er sich während der Ankunftszeit im Aufnahmezentrum aufgebaut hat, sowie Beziehungen, die er mit somalischen 
Freunden und Netzwerken virtuell pflegt, wo er auch emotionale Unterstützung erhält.

Auch wenn Ahmend auf Probleme angesprochen mit "[l]ch habe fast nie Probleme." antwortet, berichtet er - wie Aras - im Interview von Fremdheitsgefühlen und Diskriminierungserfahrungen. Gleichzeitig beschreibt er die für inn gelungene Suche und den erfolgreichen Aufbau von sozialen Unterstützungsnetzwerken, mit denen er die unterschiedlichen Herausforderungen bewältigen kann. Hierbei scheint er für sich einen Weg gefunden zu haben, mit den Fremdheitserfahrungen, dem Neuen und dem Unbekannten umgehen zu können, wie er es in der folgenden Passage erläutert:

,[l]ich bin ja immer neu für mich ist einfach normal ich bin ja immer Neuer gewesen (I: Ja.) (3) für mich ist einfach erste wo ich in Italien hingekommen bin ist für mich einfach neu gewesen aber sonst jetzt wenn irgendetwas wechselt ist für mich ganz normal (.) und ich habe jetzt auch Schule gewechselt im Fussballverein gewechselt Familie gewechselt ich wechsle alles für mich ist jetzt einfach normal weil ich lerne schnell Leute kennen." (Z. 642-648)

Deutlich macht Ahmend in dieser Passage, dass er sich einen selbstverständlichen Umgang mit Neuem angeeignet hat, der den andauernden Wechsel zum Normalzustand erklärt und auf die situative Dynamik von Agency (Eßer/Schröder 2020) verweist. Es kann vermutet werden, dass diese Haltung dadurch möglich wird, dass Ahmend spätestens seit dem Tod seiner Eltern regelmässig den Lebensmittelpunkt wechselte, mit wechselten Personenkonstellationen konfrontiert war und sein Leben weitgehend selbständig organisieren und sichern musste. Für die Herstellung von Handlungsfähigkeit innerhalb der für Ahmend oftmals häufig wechselnden Konstellationen ist es hierbei auch relevant, ob er sich auf Personen beziehen kann, die für ihn „signifikante Andere" (Göbel et al. 2020) darstellen, die ihm soziale Unterstützung, Verlässlichkeit und Kontinuität bieten.

\section{FAZIT}

Im hier präsentierten Fallvergleich zeigen sich unterschiedliche biografische Kontextualisierungen der Flucht, vor deren Hintergrund sich die verschiedenen Erlebensweisen des Ankommens in Europa deuten lassen. Für Ahmend reiht sich die Flucht nach Europa in einen Lebensentwurf ein, der durch Diskontinuität und frühe Selbständigkeit geprägt ist - und damit bereits vor der Flucht nach Europa nicht dem entsprach, was in Europa als "normale“ Kindheit gilt (Liebel 2017; Wernesjö 2012). Vor diesem Hintergrund erlebt er das Ankommen in der Schweiz zunächst als Erweiterung seiner Möglichkeitsräume und geniesst die Versorgung im Erstaufnahmezentrum. Demgegenüber erscheint die Flucht nach Europa für Aras als massiver Bruch in seinem bisherigen von Kontinuität geprägten Leben. Gegenüber den Herausforderungen und Härten, mit denen er in der Schweiz konfrontiert ist, muss er sich mühsam eine neue Haltung erarbeiten. Während für Aras der Verlust der gewohnten Sicherheit im Vordergrund steht, begreift Ahmend die Veränderungen vor allem als Gewinn.

Im Kontext ihrer weiteren Platzierung erfahren die beiden jungen Männer zunächst Fremdbestimmung und Zumutungen, die mit der Integration in der Schweiz verbunden sind. Während Aras die Zumutungen dieser Fremdbestimmung akzeptiert und auf diese Weise einen Entwicklungsprozess zu mehr Selbstbestimmung durchläuft, leistet Ahmend Widerstand gegen die ihm zugemutete Infantilisierung und erreicht eine Umplatzierung. In beiden Fällen wird jedoch kein abgeschlossener Prozess der Integration bzw. des Ankommens in der Schweiz deutlich, sondern die andauernde Notwendigkeit, sich in einer schwierigen Situation zu behaupten und zu positionieren (vgl. Lechner et. al. 2017). In beiden Fällen wird zeitweise und kontextbezogen Vulnerabilität erlebt, aber es zeigt sich auch die Eröffnung von Möglichkeitsräumen unter situativen Vulnerabilitätsbedingungen und damit deren Bewältigung. Vor diesem Hintergrund zeigen sich in der fallbezogenen Analyse weder nur Vulnerabilität (Detemple 2015; Hargasser 2016) noch ausschliesslich Agency (Heidbrinck/Statz 2017; Otto 2019), sondern es werden je eigene Kombinationen beider Aspekte deutlich. Beide Fälle sensibilisieren für eine relationale Perspektive auf 
Agency und Vulnerabilität im Kontext des Ankommensprozesses von Jugendlichen mit Fluchterfahrungen. Vulnerabilitätserfahrungen und situative Vulnerabilitätsbedingungen - wie bei Aras der Verlust seiner primären Bezugspersonen oder bei Ahmend die ihn einschränkenden und symbolisch missbrauchenden Erfahrungen in der ersten Pflegefamilie - verweisen auf begrenzende wie ermöglichende Erfahrungen im Umgang mit dem „Neuen“ und „Fremden“ (Stöhr 2019). Damit geht ein Verständnis von Agency einher, das dieses im Sinne der Eröffnung von Handlungsräumen und Handlungsmöglichkeiten versteht, die sowohl situativ im Kontext sozialer Beziehungsgefüge als auch aus einer Erfahrungsgeschichte heraus entstehen (Burkitt 2015). Die Analysen machen hierbei deutlich, dass aus Vulnerabilitätserfahrungen kontextund situationsspezifische Routinen und Strategien entwickelt werden, mit Unbekanntem umzugehen und dieses für sich zu bearbeiten. So können Haltungen entwickelt werden, die in Hinblick auf neue Erfahrungen sowohl mit dem Erleben von Begrenzungen verbunden sein können als auch mit dem Erleben grenzenloser Möglichkeitsräume. Deutlich wird hierbei auch, dass für die Herstellung einer mal durchhaltenden, mal distanzierenden, mal normalisierenden, sich in Verzicht übenden oder (ohn-)machtvollen Agency und der jeweiligen Vulnerabilitätserfahrungen auch spezifische soziale Konstellationen konstitutiv sind, um die ganz eigenen Lebensentwürfe für sich erfolgreich zu verfolgen oder darin eingeschränkt zu werden.

\section{LITERATUR}

Belloni, Milena (2019): Family project or individual choice? Exploring agency in young Eritreans' migration. In: Journal of Ethnic and Migration Studies, 46 (2), 336-353.

Burkitt, Ian (2015): Relational agency: Relational sociology, agency and interaction. In: European Journal of Social Theory, 19 (3), 322-339.

Clark, Christina R. (2007): Understanding Vulnerability: From Categories to Experiences of Young Congolese People in Uganda. In: Children and Society, 21 (4), 284-296.
Detemple, Katharina (2016): Zwischen Autonomie und Hilfebedarf. Unbegleitete minderjährige Flüchtlinge in der Jugendhilfe. Baltmannsweiler: Schneider Verlag Hohengehren.

Eßer, Florian/Schröder, Christian (2020): Agency und Vulnerabilität im Care Leaving. Ein Kommentar. In: Göbel, Sabrina/Karl, Ute/Lunz, Marei/Peters, Ulla/Zeller, Maren (Hrsg.): Wege junger Menschen aus Heimen und Pflegefamilien. Agency in schwierigen Übergängen. Weinheim: Beltz Juventa, 292-307.

Findening, Ines/Klinger, Sabine (2019): Ambivalente Lebenswelten. Unbegleitete Minderjährige mit Fluchterfahrung in der österreichischen stationären Kinder- und Jugendhilfe. In: Zeitschrift für Migration und Soziale Arbeit, 41 (4), 368-374.

Göbel, Sabrina/Hadjar, Andreas/Karl, Ute/Peters, Ulla/Jäger, Julia A. (2020): Bildungsverläufe und Agency von Care Leaver_innen in Luxemburg. In: Göbel, Sabrina/Karl, Ute/Lunz, Marei/Peters, Ulla/Zeller, Maren (Hrsg.): Wege junger Menschen aus Heimen und Pflegefamilien. Agency in schwierigen Übergängen. Weinheim: Beltz Juventa, 83-104.

Hargasser, Brigitte (2016): Unbegleitete minderjährige Flüchtlinge. Sequentielle Traumatisierungsprozesse und die Aufgabe der Jugendhilfe. Frankfurt am Main: Brandes \& Apel.

Heidbrinck, Lauren/Statz, Michael (2017): Parents of global youth: contesting debt and belonging. In: Children's Geographies, 15 (5), 545-557.

Helfferich Cornelia (2020): Rekonstruktive Agency-Analyse in der Forschung zu Leaving Care. Methodologischer Hintergrund, praktisches Vorgehen und ergiebige Fragestellungen. In: Göbel, Sabrina/Karl, Ute/Lunz, Marei/Peters, Ulla/Zeller, Maren (Hrsg.): Wege junger Menschen aus Heimen und Pflegefamilien. Agency in schwierigen Übergängen. Weinheim: Beltz Juventa, 50-67.

Jurt, Luzia/Roulin, Christophe (2016): Begleitung und Betreuung von unbegleiteten minderjährigen Asylsuchenden: Die Wahrnehmung von 
Care-Arbeit aus Sicht der Klientinnen und Klienten. In: Diskurs Kindheits- und Jugendforschung, 11 (1), 99-111.

Kurz-Adam, Maria (2016): Kinder auf der Flucht. Die soziale Arbeit muss umdenken. Opladen: Barbara Budrich.

Lechner, Claudia/Huber, Anna/Holthusen, Bernd (2017): „Familie, Schule, Freunde - Ich wünsche mir ein ganz normales Leben!" - Die Sicht begleiteter und unbegleiteter junger Geflüchteter auf ihre Lebenslagen. In: Jugendhilfe, 55 (1), 11-19.

Lems, Annika/Oester, Kathrin/Strasser, Sabine (2019): Children of the crisis: ethnographic perspectives on unaccompanied refugee youth in and en route to Europe. In: Journal of Ethnic and Migration Studies, 46 (2), 315-335.

Liebel, Manfred (2017): Postkoloniale Kindheiten: zwischen Ausgrenzung und Widerstand, Weinheim: Beltz Juventa.

Mackenzie, Catriona/Rogers, Wendy/Dodds, Susan (2013): Vulnerability. New Essays in Ethics and Feminist Philosophy. Oxford/New York: Oxford University Press.

Magyar-Haas, Veronika (2017): Ausgesetzter Körper, verletzbarer Leib. Zur Regulierbarkeit der Emotionen. In: Zeitschrift für Soziologie der Erziehung und Sozialisation, 37 (1), 39-54.

Niggli-Gamper, Anouk (2019): Ankommen in einer neuen Welt. Unbegleitete minderjährige Geflüchtete in Schweizer Pflegefamilien. Zürich: unveröffentlichte Masterarbeit.

Otto, Laura (2019): Children, adults or both? Negotiating adult minors and interests in a state care facility in Malta. In: Journal of Ethnic and Migration Studies, 46 (2), 372-388.

Payne, Ruth (2012): 'Extraordinary survivors' or 'ordinary lives'? Embracing 'everyday agency' in social interventions with child-headed households in Zambia. In: Children's Geographies, 10 (4), 399-411.
Rieker, Peter/Höhne, Ellen/Mörgen, Rebecca (2021): Unterbringung und Betreuung unbegleiteter minderjähriger Geflüchteter in der Schweiz aus Sicht von Fachpersonen. In: Schweizerische Zeitschrift für Soziale Arbeit, 9-30.

Stöhr, Robert (2019): Zwischen Pathos und Response. Bernhard Waldenfels über Verletzlichkeit als Grundmoment der Erfahrung. In: Stöhr, Robert/Lohwasser, Diana/Napoles, Juliane Noack/Burghardt, Daniel/Dederich, Markus/Dziabel, Nadine/Krebs, Moritz/Zirfas, Jörg (Hrsg.): Schlüsselwerke der Vulnerabilitätsforschung. Wiesbaden: Springer VS Verlag, 145-167.

Strauss, Anselm (1998): Grundlagen qualitativer Sozialforschung. München: Wilhelm Fink Verlag.

Utas, Mats (2005): Victimcy, Girlfriending, Soldiering: Tactic Agency in a Young Woman's Social Navigation of the Liberian War Zone. In: Anthropological Quarterly, 78 (2), 403-430.

Waldenfels, Bernhard (2002): Bruchlinien der Erfahrung. Frankfurt am Main: Suhrkamp.

Wernesjö, Ulrika (2012): Unaccompanied Asylum-Seeking Children: Whose Perspective? In: Childhood, 19 (4), 495-507.

Witzel, Andreas (2000): Das problemzentrierte Interview [25 Absätze]. In: Forum Qualitative Sozialforschung 1(1), https://www.qualitative-research.net/index.php/fas/article/view/1132 (04.03.2021).

Zeller, Maren/Köngeter, Stefan/Meier, Leonie (2020): Vertrauen und Zukunftsvorstellungen bei jungen Geflüchteten im Übergang. In: Göbel, Sabrina/Karl, Ute/Lunz, Marei/Peters, Ulla/Zeller, Maren (Hrsg.): Wege junger Menschen aus Heimen und Pflegefamilien. Agency in schwierigen Übergängen. Weinheim: Beltz Juventa, 204224. 


\section{Zu den Autor*innen}

Rebecca Mörgen arbeitet als wissenschaftliche Mitarbeiterin (Postdoc) am Lehrstuhl für Ausserschulische Bildung und Erziehung am Institut für Erziehungswissenschaft der Universität Zürich. Ihre Forschungsund Interessensschwerpunkte liegen in den Bereichen der qualitativ-empirischen Migrations-, Ungleichheits- und Professionsforschung im Kontext Sozialpädagogik. Zudem beschäftigt sie sich mit Fragen sozialer Teilhabemöglichkeiten und arbeitet zu Methoden der qualitativen Sozialforschung, insbesondere Ethnographie, partizipative Ansätze.

Peter Rieker leitet den Lehrstuhl für Ausserschulische Bildung und Erziehung am Institut für Erziehungswissenschaft der Universität Zürich. Er forscht zu Kindheit und Jugend, zu abweichendem Verhalten und Extremismus sowie zu politischer Partizipation, Migration und interethnischen Beziehungen. Zudem arbeitet er zu Methoden der qualitativen Sozialforschung und zu multimethodischer Forschung.

\section{Kontakt}

Dr. Rebecca Mörgen

Institut für Erziehungswissenschaft

Universität Zürich

Freiestr. 36

$\mathrm{CH}-8032$ Zürich

Tel.: +41446344563

E-Mail: rmoergen@ife.uzh.ch

URL: https://www.ife.uzh.ch/de/research/abe/mitarbeitende2/moergenrebecca.html

Prof. Dr. Peter Rieker

Institut für Erziehungswissenschaft

Universität Zürich

Freiestr. 36

$\mathrm{CH}-8032$ Zürich

Tel.: +41446344561

E-Mail: prieker@ife.uzh.ch

URL: https://www.ife.uzh.ch/de/research/abe/mitarbeitende2/riekerpeter.html 